EESTI NSV TEADUSTE AKADEEMIA TOIMETISED. XII KÖIDE FOOSIKA-MATEMAATIKA- JA TEHNIKATEADUSTE SEERIA. 1963, NR. 2

ИЗВЕСТИЯ АКАДЕМИИ НАУК ЭСТОНСКОИ ССР. ТОМ ХІІ СЕРИЯ ФИЗИКО-МАТЕМАТИЧЕСКИХ И ТЕХНИЧЕСКИХ НАУК. 1963, № 2

\title{
К ВОПРОСУ ОПРЕДЕЛЕНИЯ МЕТОДИЧЕСКОЙ ПОГРЕШНОСТИ ЛИНЕЙНОГО ДЕТЕКТОРА ЭФФЕКТИВНЫХ ЗНАЧЕНИИ СО СКОЛЬЗЯЩИМ СМЕЩЕНИЕМ
}

\author{
л. и. волгин
}

Известно, что зависимость показаний линейного детектора эффективных значений со скользящим смещением и линейно-сегментной аппроксимацией вольтамперной характеристики (ЛДЭЗ) от формы кривой измеряемого напряжения не превосходит величины порядка нескольких процентов при коэффициенте амплитуды измеряемого напряжения $K_{a}$, не превышающем пяти $[1,2,3]$. При этом существует мнение [3], что зависимость показаний ЛДӘЗ от формы кривой измеряемого напряжения полностыю определяется погрешностью аппроксимации. Для уточнения этого положения необходимо провести более строгий анализ работы ПДӘЗ.

На рис. 1 приведена схема ЛДЭ3, работающая в режиме заданного напряжения.

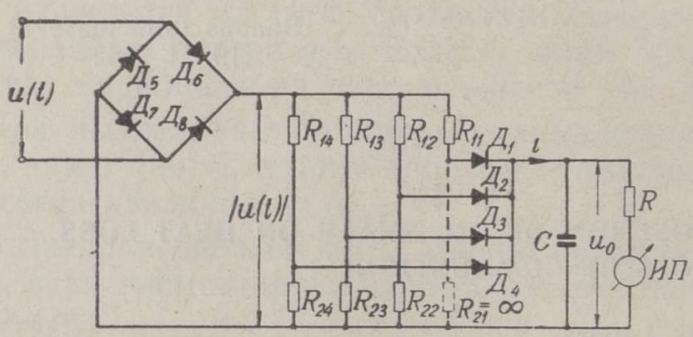

Рис. 1. Схема ЛДЭЗ, работающая в режиме заданного напряжения.

На диодах $Д_{5}, Д_{6}, Д_{7}$ и $Д_{8}$ собран двухполупериодный линейный выпрямитель. C помощью диодов Д $i(i=1,2,3,4)$ и делителей напряжения $R_{1 i}-$ $R_{2 i}$ аппроксимируется параболическая зависимость

$$
\frac{i}{u_{0}}=b\left(\frac{u}{u_{0}}\right)^{2}
$$

где $b$ - числовой коэффициент.

(Во избежание многозначности при графическом изображении вольтамперной характеристики зависимость (1) нормирована по напряжению $u_{0}$.) Реальная вольтамперная характеристика (см. рис. 2)

$$
\frac{i}{u_{0}}=f\left(\frac{u}{u_{0}}\right)
$$

при $\frac{u}{u_{0}}=1, \frac{u}{u_{0}}=\frac{R_{1 i}+R_{2 i}}{R_{2 i}}$ имеет точки перегиба. 
Рис. 2. Вольтамперная характеристика ЛдЭ3, нормированная по напряжению $u_{0}(t)$.

Выражение (1) аппроксимирует реальную вольтамперную характеристику с заданной степенью точности при условии, что $0 \leqslant \tau \leqslant \tau_{\text {кр }}$ (где $\tau=R C$ есть постоянная времени разрядной цепи). В дальнейшем изложении будем предполагать, что значение $\tau$ не превосходит величины $\tau_{\mathrm{kp}}$.

Угол отклонения стрелки индикаторного прибора магнито-электрической системы (ИП) линейно зависит от значения $u_{0}$, т. е. вольтамперная характеристика $i=F(u)$ в зависимости от величины $u_{0}$ сдвигается параллельно самой себе

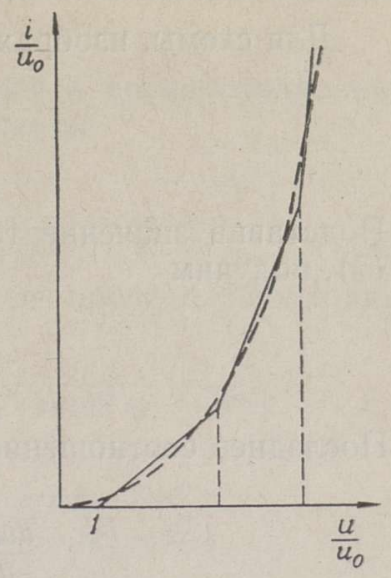
в направлении оси абсцисс.

Для установившегося режнма в первом приближении можно считать, что $u_{0}=$ const, тогда имеем

$$
\frac{1}{T} \int_{0}^{T} i d t=\frac{u_{0}}{R}
$$

С учетом условия (1) из соотношения (3) находим

$$
u_{0}=U_{\ni} \sqrt{b R}
$$

где

$$
U_{\ni}=\sqrt{\frac{1}{T} \int_{0}^{T} t t^{2} d t}
$$

Равенство (4) показывает, что шкала прибора имеет линейный характер. Это является несомненным достоинством данного способа детектирования, так как наличие квадратичной шкалы приводит к повышенным величинам допустимых относительных погрешностей измерения [ $\left.{ }^{4}\right]$.

При выводе соотношения (4) были сделаны следующие допущения: вольтамперная характеристика детектора, нормированная по величине $u_{0}$, является идеальной параболой, определяемой выражением (1); в установившемся режиме $u_{0}=$ const.

Последнее предположение является довольно грубым допущением, так как мгновенное значение постоянной времени заряда зависит от мгновенного значения измеряемого напряжения, именно эта зависимость обусловливает возможность измерения эффективного значения напряжений произвольной формы.

При более строгой постановке вопроса, считая, что $u_{0}(t)=\mathrm{var}$, необходимо найти величину

$$
U_{0}=\frac{1}{T} \int_{0}^{T} u_{0}(t) d t
$$

так как угол отклонения стрелки прибора пропорционален значению $U_{0 \text { - }}$ 
Для схемы, изображенной на рис. 1, можно записать

$$
i=c \frac{d u_{0}(t)}{d t}+\frac{u_{0}(t)}{R}
$$

Подставив значение тока, определяемое выражением (1), в равенство (7), получим

$$
\frac{b u^{2}(t)}{u_{0}(t)}=c \frac{d u_{0}(t)}{d t}+\frac{u_{0}(t)}{R}
$$

Последнее соотношение можно переписать в следующем виде

$$
\frac{d u_{0}^{2}(t)}{d t}+\frac{2}{\tau} u_{0}^{2}(t)=\frac{2 b R}{\tau} u^{2}(t)
$$

Это дифференциальное уравнение первого порядка линейное относительно $u_{0}^{2}(t)$. Решение уравнения можно представить в следующем виде

$$
u_{0}(t)=\sqrt{\frac{2 b R}{\tau}\left[e^{-2 t / \tau} \int u^{2}(t) e^{2 t / \tau} d t+A e^{-2 t / \tau}\right]}
$$

где $A-$ постоянная интегрирования.

B установившемся режиме при достаточно большом $t$ слагаемое $A e^{-2 t \tau}$ равно нулю. С учетом равенства (6) окончательно получим

$$
U_{0}=\frac{1}{T} \int_{0}^{T} \sqrt{\frac{2 b R}{\tau} e^{-2 t / \tau} \int u^{2}(t) e^{2 t / \tau} d t} d t
$$

Найдем значение $U_{0}$, определяемое выражением $(10)$, для некоторых конкретных видов измеряемого напряжения.

\section{1. Напряжение вида «квадратная волна»}

В соответствии с равенством (10), проведя несложные вычисления, получим

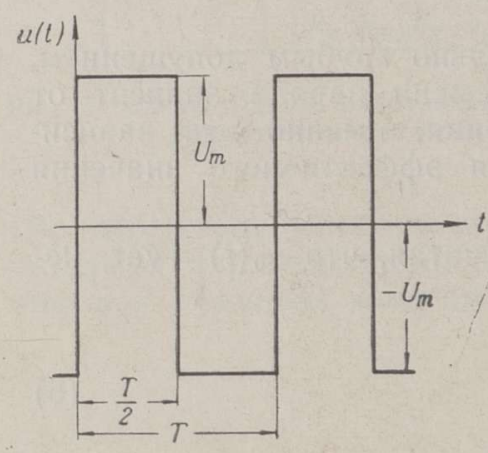

Рис. 3. Напряжение вида «квадратная волна».

$$
U_{0}=U_{m} \sqrt{b R}=U_{\ni} \sqrt{b R}
$$

что совпадает с выражением (4). Таким образом, в данном случае, исходя из допущения, что вольтамперная характеристика детектора является идеальной параболой, погрешность измерения эффективного значения напряжения отсутствует. В дальнейшем будем предполагать, что детектор отградуирован при напряжении вида «квадратная волна». 


\section{2. Напряжение, состоящее из синусоидальной и косинусоидальной составляющих равной частоты}

$$
u(t)=U_{m} \sin \omega t+U_{m_{1}} \cos \omega t=U_{m}(\sin \omega t+n \cos \omega t)
$$

Подставив в равенство (9) значение $u(t)$ и проведя необходимые операции, найдем

$$
u_{0}(t)=\sqrt{0,5 b R\left(1+n^{2}\right) U_{m}^{2}\left[1-\sin \varphi_{2} \cdot \sin (2 \omega t+\Psi)\right]}
$$

где

$$
\sin \varphi_{2}=\frac{1}{\sqrt{1+\omega^{2} \tau^{2}}}, \quad \Psi=\operatorname{arctg}\left[\frac{1+2 \omega \tau n-n^{2}}{\omega \tau\left(1-n^{2}\right)-2 n}\right]
$$

Проведя разложение последнего соотношения в ряд, в соответствии с формулой (10), получим

где

$$
U_{0}=U_{\ni} \sqrt{b R}\left(1+\delta \cdot 10^{-2}\right),
$$

$$
U_{9}=U_{m} \sqrt{0,5\left(1+n^{2}\right)} \text {; }
$$

$\delta$ - относительная погрешность, выраженная в процентах, обусловленная рассматриваемым способом детектирования (методическая погрешность), причем

$$
\begin{array}{r}
\delta=\sum_{k=1}^{\infty}\left[\frac{B_{k}}{\left(1+\omega^{2} \tau^{2}\right)^{k}}\right] \cdot 100 \%, \\
B_{k}=\frac{1}{(2 k) !} \prod_{i=0}^{2 k-1}(0,5-i) \cdot \prod_{l=1}^{k}\left(1-\frac{1}{2 l}\right) .
\end{array}
$$

Ряд (12) является сходящимся для всех значений $\omega \tau$. При $k=1$ равенство (12) с учетом выражения (13) перепишется в следующем виде

$$
\delta=-\frac{100 \%}{16\left(1+\omega^{2} \tau^{2}\right)} .
$$

Последнее соотношение справедливо при $\tau \gg T$, т. е. при выполнении условия $\sin \varphi_{2} \cdot \sin (2 \omega t+\Psi) \ll 1$. Для значений $\omega \tau<2$ формула (14) дает довольно грубое приближение, так как постоянная времени разрядной цепи становится соизмеримой с периодом $T$. Действительно, в наихудшем случае при $\tau=0$ (детектор средних значений) величину погрешности, обусловленную отклонением формы кривой измеряемого напряжения от градуировочного, можно найти из следующего известного соотношения

$$
\delta=\left(\frac{K_{\phi 0}}{K_{\phi}}-1\right) \cdot 100 \%
$$

тде $K_{\phi 0}-$ коэффициент формы для напряжения, на котором произведена градуировка;

$K_{\Phi}$ - коэффициент формы измеряемого напряжения. 
В рассматриваемом случае для напряжения вида «квадратная волна» $K_{\phi 0}=1$, для синусоидального напряжения $(n=0) K_{\phi}=1,1107$. Подставив указанные значения в выражение (15), найдем, что $\delta=-9,967 \%$. Между тем. из выражения (14) для $\tau=0$ находим, что $\delta=-6,25 \%$. Соответствующая величина погрешности для $\tau=0$, вычисленная по формуле (12), при суммировании первых двадцати слагаемых равна -9,916\%, что почти совпадает со значением, полученным из выражения (15).

На рис. 4 пунктиром изображена зависимость методической погрешности от величины $\omega \tau$, вычисленная по формуле (14), соответствующая зависимость, вычисленная по формуле (12) для первых двадцати членов, изображена сплошной линией.

\section{3. Напряжение, состоящее из основной и второй гармоник}

$$
u(t)=U_{m} \sin \omega t+U_{m 1} \sin 2 \omega t=U_{m}(\sin \omega t+n \sin 2 \omega t)
$$

Подставив в равенство (9) значение $u(t)$ и проведя необходимые операции, найдем

$$
\begin{gathered}
u_{0}(t)=\sqrt{0,5 b R\left(1+n^{2}\right) U_{m}^{2}\left[1-\frac{n^{2} \sin \varphi_{4} \cdot \sin \left(4 \omega t+\varphi_{4}\right)}{1+n^{2}}-\right.} \\
\left.-\frac{2 n \sin \varphi_{3} \cdot \sin \left(3 \omega t+\varphi_{3}\right)}{1+n^{2}}-\frac{\sin \varphi_{2} \cdot \sin \left(2 \omega t+\varphi_{2}\right)}{1+n^{2}}+\frac{2 n \sin \varphi_{1} \cdot \sin \left(\omega t+\varphi_{1}\right)}{1+n^{2}}\right]
\end{gathered}
$$

где

$$
\begin{array}{ll}
\sin \varphi_{1}=\frac{1}{\sqrt{1+4 \omega^{2} \tau^{2}}}, . & \sin \varphi_{3}=\frac{1}{\sqrt{1+2,25 \omega^{2} \tau^{2}}}, \\
\sin \varphi_{2}=\frac{1}{\sqrt{1+\omega^{2} \tau^{2}}}, & \sin \varphi_{1}=\frac{1}{\sqrt{1+0,25 \omega^{2} \tau^{2}}} .
\end{array}
$$

При $\tau \gg T(T-$ период основной гармоники), проведя разложение последнего соотношения в ряд, пренебрегая членами третьего и более высших порядков малости, в соответствии с формулой (10) получим соотношение вида (11), в котором значение $\delta$ определяется следующим выражением

$$
\delta=-\frac{100 \%}{16\left(1+n^{2}\right)^{2}}\left(\frac{n^{4}}{1+4 \omega^{2} \tau^{2}}+\frac{4 n^{2}}{1+2,25 \omega^{2} \tau^{2}}+\frac{4 n^{2}}{1+0,25 \omega^{2} \tau^{2}}+\frac{1}{1+\omega^{2} \tau^{2}}\right) .
$$

Заметим, что при $n=0$ (синусоидальное напряжение) выражение (16) совпадает с формулой (14).

На рис. 4 штрих-пунктирной линией изображена зависимость методической погрешности от величины $\omega \tau$, вычисленная по формуле (16). для $n=1$ (коэффициент амплитуды $\left.K_{a}=\sqrt{3}\right)$.

Таким образом, проведенный анализ позволяет сделать вывод, что зависимость показаний ЛДЭЗ от формы кривой измеряемого напряжения обусловлена не только погрешностью аппроксимации квадратичной зависимости (1), но также и погрешностью данного способа детектирования, т. е. существует методическая погрешность, величина которой зависит от формы кривой измеряемого напряжения и от величины $\omega \tau$. 
При заданном наибольшем значении коэффициента амплитуды указанная погрешность не превосходит определенной величины.

На рис. 5 приведена схема ЛДЭ3, работающая в режиме заданного тока [3]. В соответствии с выражением (10) эквивалентную блок-схему для обоих типов ЛДЭЗ можно представить в следующем виде (см. рис. 6).

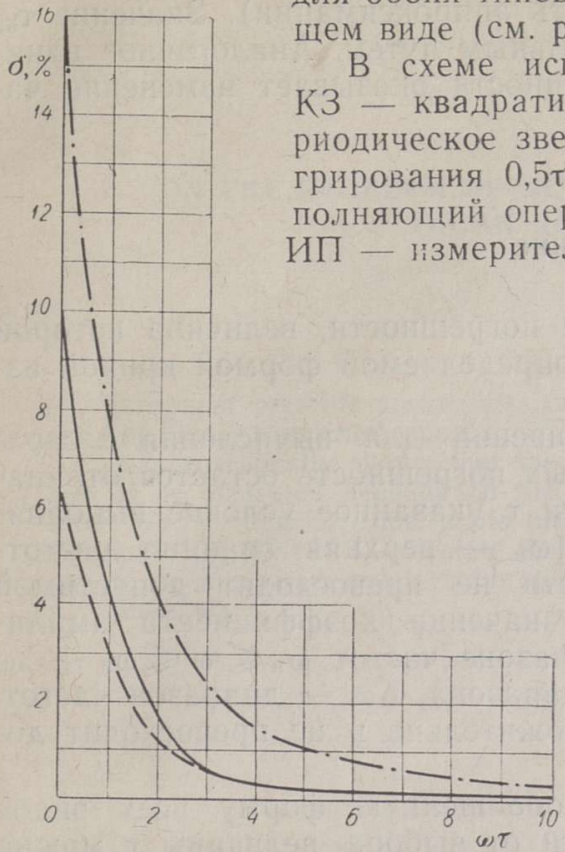

Рис. 4. Зависимость методической погрешности от величины $\omega \tau$.

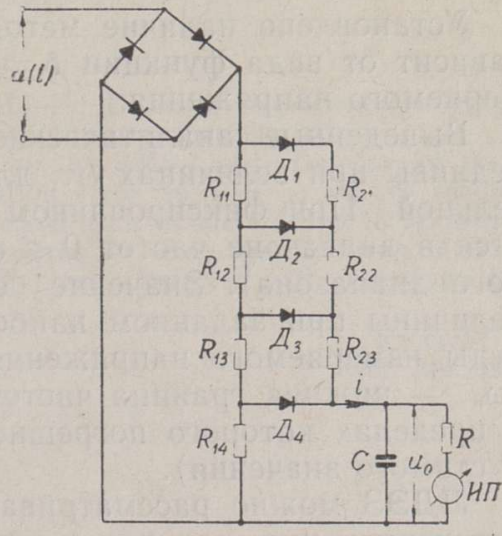

Рис. 5. Схема ЛДЭЗ, работающая в режиме заданного тока.

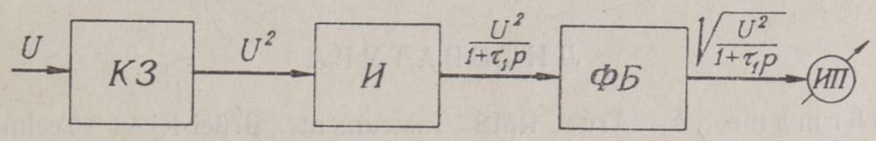

Рис. 6. Эквивалентная блок-схема ЛДЭЗ.

Как уже отмечалось, проведенный анализ справедлив для значений $\tau<\tau_{\text {кр }}$ при заданном диапазоне частот измеряемых напряжений, так как в предельном случае при $\tau \rightarrow \infty$ ЛДЭЗ превращается в пиковый детектор, т. е. условие (1) теряет смысл. При увеличении $\tau$ от нуля до $\tau_{\text {кр }}$ погрешность уменьшается и при $\tau=\tau_{\text {кр }}$ равна нулю и при дальнейшем увеличении $\tau$, меняя знак, резко возрастает (имеется в виду определенная форма кривой измеряемого напряжения при фиксированной частоте). Согласно известному соотношению при пиковом детектировании $(\tau \rightarrow \infty)$

$$
\delta=\left(\frac{K_{a}}{K_{a 0}}-1\right) \cdot 100 \%
$$

где $K_{a 0}-$ коэффициент амплитуды напряжения, на котором произведена градуировка прибора (в нашем случае $\left.K_{a 0}=1\right), K_{a}-$ коэффициент амплитуды измеряемого напряжения, т. е. для случая синусои- 
дального напряжения $\left(K_{a}=\sqrt{2}\right) \delta=+41 \%$. Для различных форм кривых измеряемого напряжения значения $\tau_{\text {кр }}$ не совпадают, но располо жены близко друг от друга, что позволяет, например, для напряжений с $K_{a} \leqslant 5$ получить величину погрешности, не превосходяшую $5 \%$ (в указанную величину входит и погрешность аппроксимации). Значение $\tau_{\mathrm{kp}}$ не трудно определить и экспериментальным путем. Аналогичное влия ние на величину методической погрешности оказывает изменение частоты измеряемого напряжения.

\title{
Заключение
}

Установлено наличие методической погрешности, величина которой зависит от вида функции $\delta=\varphi(\omega t)$, определяемой формой кривой измеряемого напряжения.

Выведенные аналитические соотношения для вычисления $\delta$ справедливы при величинах $\omega \tau$, для которых погрешность остается отрицательной. При фиксированном значении $\tau$ указанное условие выполня ется в диапазоне частот $0 \leqslant \omega \leqslant \omega_{\text {в }}\left(\omega_{\text {в }}\right.$ - верхняя граница частотного диапазона). Значение погрешности не превосходит допустимой величины при заданном наибольшем значении коэффициента амплитуды измеряемого напряжения в диапазоне частот $\omega_{\text {н }} \leqslant \omega \leqslant \omega_{\text {в }}+\Delta \omega$ $\left(\omega_{\text {н }}\right.$ - нижняя граница частотного диапазона, $\Delta \omega-$ диапазон частот, в пределах которого погрешность положительна и не превосходит допустимого значения).

ЛДЭЗ можно рассматривать как обобщенную форму всех видов детектирования, так как в зависимости от выбора величины $\tau$ можно получить любой вид детектирования, в частности, линейный детектор, детектор эффективных значений и ликовый детектор $\left(\tau=0, \tau_{\mathrm{kp}}, \infty\right.$ соответственно) .

\section{Л ИТ Е РА Т УР А}

1. C. G. Wahrmann, A True RMS Instrument. Brüel-Kjaer Technical Review, № $3,1958$.

2. Effektivwert-Gleichrichter für Drehspulinstrumente. Elektronische Rundschau, № 4,1961

3. E. So n n t a g, Effektivwertmesser mit gestaffelt vorgespannten Dioden und gleitender Knickspannung. Hochfrequenztechnik und Elektroakustik, Bd. 70, ViI, № 3, 196!.

4. Л. И. В олг и н, Об оценке погрешностей вдоль квадратичных шкал измерительных приборов со стрелочным указателем. Вопросы радиоэлектроники, сер. XI, вып. 3, 1961.

Поступила в редакцию

4. X 1962

\section{MUUTUVA EELPINGEGA EFEKTIIVVAARTUSE LINEAARSE DETEKTORI METOODILISE VEA MÄARAMISEST}

\author{
L. Volgin \\ Resümee
}

Artiklis esitatakse muutuva eelpingega efektiivväärtuse lineaarse detektori (ELD) töö analüüs, milles kasutatakse voltampertunnusjoone lineaar-segment aproksimeerimist. Analüütiliselt tõestatakse, et ELD näidu sōltuvus mõōdetava pingekõvera kujust on tingi- 
tud mitte ainult aproksimeerimise veast, vaid ka metoodilisest veast, mille suurus sõltub mõõdetava pingekõvera kujust. Tuletatakse valemid metoodilise vea väljaarvutamiseks mõningate konkreetsete pingekujude jaoks (kui ELD kalibreeritakse sümmeetrilise püstkülikukujulise pingega).

Saabus toimetusse

4. X 1962

\section{ON THE PROBLEM OF DETERMINING THE METHODICAL ERROR OF AN RMS-LINEAR DETECTOR}

\section{Volgin \\ Summary}

The paper presents an analysis of an RMS-Linear Detector employing a sliding bias and the linear-segmental approximation of its volt-ampere characteristic.

It is analytically shown that the recording by the RMS-Linear Detector of the voltage to be measured depends on the approximation error as well as on the methodical error, whose value depends, in its turn, on the wave form of the voltage to be measured. Formulas are derived to calculate the methodical error for some specific forms of the voltage to be measured (while calibrating the RMS-Linear Detector with a voltage having a "square wave").

Received

Oct. 4th, 1962 